\title{
SiNGLE CRYSTAL MAGNESIUM LITHIUM ALLOY ELASTIC CONSTANTS
}

\author{
Nataliya Shkatulyak ${ }^{1}$, Valentin Usov $^{2}$ and Svetlana Smirnova ${ }^{1}$ \\ ${ }^{1}$ Department of Physics, South-Ukrainian National Pedagogical University named after \\ K.D. Ushinsky, 26 Staroportofrankovskaya Str., 65020, Odessa, Ukraine \\ ${ }^{2}$ Head of Department of technological and professional education, South-Ukrainian \\ National Pedagogical University named after K.D. Ushinsky, 26 Staroportofrankovskaya \\ Str., 65020, Odessa, Ukraine
}

\begin{abstract}
Knowledge of elastic characteristics of the structural materials such as elastic modules, allows to develop constructions that resulting deformation are foreseeable and not excessive in terms of plastic deformation, accelerated fatigue or even fracture. In this paper we investigate the elastic properties of the binary alloy Mg-5\% Li (mass.). For measuring of single crystal elastic constants of alloy is needed to have the crystal of relatively large size. For the alloy this is connected with the technical difficulties. In this paper we propose to determine the elastic constants of single crystals of alloy magnesium-lithium using the experimental anisotropy of Young's modulus and integral characteristics of texture (ICT), which can be found from the pole figure \{0002\}. We have calculated the anisotropy of Young's modulus alloy sheet Mg5\% Li using found magnesium-lithium single crystal constants. Deviation of calculated values from experimental did not exceed $5 \%$ that is not bad result.
\end{abstract}

\section{KEYWORDS}

Elastic Constants, Single Crystals, Magnesium-Lithium Alloy, Integral Characteristics of Texture, Elastic Anisotropy, Polycrystalline Sheet

\section{List of symbols}

$\varepsilon_{i j}, \varepsilon_{i}$ are components of deformations second-rank tensor;

$\sigma_{i j}, \sigma_{i}$ are components of stresses second-rank tensor;

$S_{i j k l}, S_{i j}$ are components of the fourth rank of elastic compliances tensor

$C_{i j k l}, C_{i j}$ are components of the fourth rank of elastic stiffness's (modules) tensor

$E$ is the Young's modulus

$G$ is the shear modulus

$I_{i}$ are integral characteristic of texture

$\Sigma_{1}(\gamma)$ and $\Sigma_{2}(\gamma)$ are functions of texture sheet

\section{INTRODUCTION}

For the last time it was established a good enough biocompatibility and biodegradability of binary alloys $\mathrm{Mg}$-Li. For example, are known biodegradable alloys $\mathrm{Mg}-3.5 \mathrm{Li}, \mathrm{Mg}-8.5 \mathrm{Li}$ and $\mathrm{Mg}-14 \mathrm{Li}$ (wt. \%). Double magnesium lithium alloys relatively easily undergo formability. The above DOI : 10.14810/ijamse.2015.4401 
mentioned properties together with not high modulus of elasticity, and high corrosion resistance make binary magnesium-lithium alloys promising for use in bio-medical technologies [1].

The high specific strength combined with low density magnesium lithium alloys makes them attractive to use in the aerospace and transportation industries [2]. However, widespread adoption in the production of magnesium lithium alloys prevents lack of knowledge on the effect of texture on the anisotropy, in particular, of elastic properties and formability at external influences.

As is known, a homogeneous deformations and stresses in general case are second-rank tensors. $[3,4]$. Therefore, Hooke's law in a generalized form for crystals can be written as

$$
\begin{gathered}
\varepsilon_{i j}=S_{i j k l} \sigma_{k l}, \\
\sigma_{i j}=C_{i j k l} \varepsilon_{k l} .
\end{gathered}
$$

Where $S_{i j k l}$ and $C_{i j k l}$ are components of the symmetrical fourth rank tensor. Coefficients $S_{i j k l}$ are elastic compliances. They have the dimension of the inverse stress $\mathrm{m}^{2} / \mathrm{N}$. Coefficients $C_{i j k l}$ are elastic stiffness's (modules). They have the dimension of stress $\mathrm{N} / \mathrm{m}^{2}$. Values $\varepsilon_{\mathrm{ij}}$ are dimensionless quantities.

The recording is simplified in matrix notation, by replacing the first two indexes on the one index that varies from 1 to $6[3,4]$. A similar substitution is applied to two last indexes. Then (1) and (2) take the form

$$
\begin{aligned}
& \varepsilon_{i}=S_{i j} \sigma_{j}(\mathrm{i}, \mathrm{j}=1,2, \ldots, 6), \\
& \sigma_{i}=C_{i j} \varepsilon_{j}(\mathrm{i}, \mathrm{j}=1,2, \ldots, 6) .
\end{aligned}
$$

Here $\left(S_{i j}\right)$ and $\left(C_{i j}\right)$ are matrices with six rows and six columns. Than the crystal symmetry is higher, the smaller the number of independent components of the elasticity tensor. For example, a hexagonal crystal is characterized by five linearly independent components, and cubic crystal has only three.

The physical meaning of the elastic constants becomes clear by examining the basic elementary types of stress state of an elastic body.

For example, the elastic constant $C_{11}$ corresponds to the Young's modulus $E$ under the simple longitudinal stretching (compression), that is, $C_{11}=E$ or $S_{11}=1 / E$. In the case of pure shear the component $C_{44}$ of the elasticity tensor or the inverse value component of elastic compliances tensor $1 / S_{44}$ have a sense of the shear modulus $G$.

In general case, the crystalline rod under the effect of a simple tensile acquires longitudinal distension, the transverse compression and shear deformation. A more detailed description of the physical meaning of the elastic constants of the crystal can be found in [3, 4].

The calculation of structures made of metal alloys requires knowledge of elastic constants of these alloys instead their values for the pure metal, on the base of which are obtained these alloys. Otherwise, the calculations may be wrong. Typically, the elastic constants are measured on single crystals of relatively large size. But the production of single crystals alloy of the right size for measuring often it represents an independent, quite a difficult task.

Previously we have found some combinations of elastic constants of single crystal of alloy ZE10 (alloy of magnesium with zinc, zirconium and rare earth metals) from the anisotropy measurement of Young's module in the polycrystalline sheet of alloy and integral characteristics of texture (ICT) [5]. 
The present article is aimed at solving a similar problem for the alloy $\mathrm{Mg}-5 \% \mathrm{Li}$ (mass.).

\section{MATERIALS AND METHODS}

\subsection{Materials}

The magnesium alloy sheet containing $5 \%$ of Li (mass) was used as material for studies. Before study, the alloy was extruded at $380^{\circ} \mathrm{C}$ [6] through a rectangular die for obtaining of sheet of thickness of $4 \mathrm{~mm}$ and a width of $120 \mathrm{~mm}$.

We have cut from sheet of the studied alloy in different direction 7 rectangular specimens, in order to determine experimentally the anisotropy of Young's modulus. Samples had a length of $100 \mathrm{~mm}$ and a width of $10 \mathrm{~mm}$. We have cut them through every 15 degrees. The first sample was oriented in the direction of extrusion ED. The last, a seventh sample, was cut in the transverse direction TD (Figure 1).

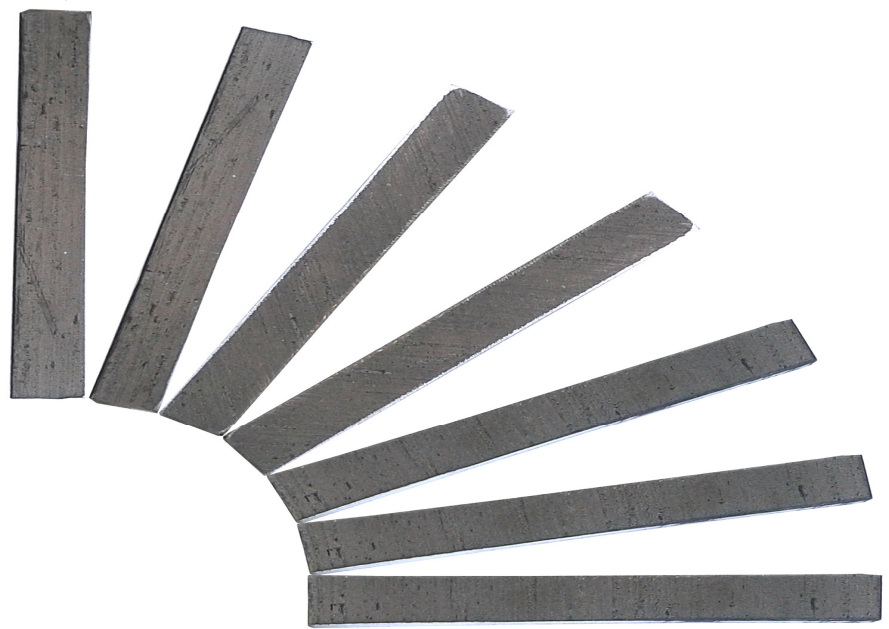

Figure 1. Photo of samples, cut from a sheet alloy $\mathrm{Mg}-5 \% \mathrm{Li}$ to measure the anisotropy of Young's modulus

\subsection{Methods}

Samples were processed in the packet to reduce measurement errors due to geometrical dimensions. Equipment scheme for measuring of Young's modulus is shown on the Figure 2. The error did not exceed $1 \%$.

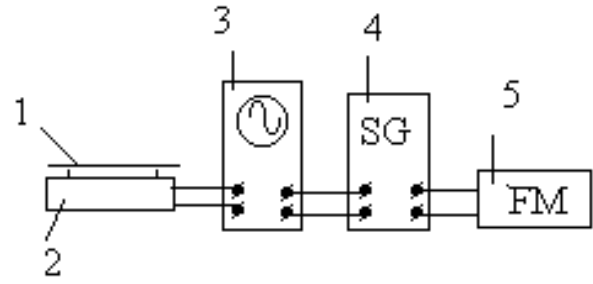

Figure 2. Equipment scheme for measuring of Young's modulus

The sample 1 rests on the elastic filaments near the microphone 2. Free transverse mechanical vibrations in the sample 1 are excited by a slight bump of the graphite stick. Electrical oscillations that occur in the microphone under the influence of mechanical vibrations sample are transmitted for vertical plates of oscilloscope 3. On his horizontal plates is transmitted the electrical signal from the sound generator (SG) 4. On the oscilloscope screen are summed two perpendicular oscillations (of microphone and SG). If frequency signals coincide, ellipse appears on the screen. 
The oscillation frequency is measured by frequency meter (FM) 5. Young's modulus for prismatic sample is calculated by means of relation [7]

$$
E=0.968 \rho \frac{l^{4}}{d^{2}} v^{2},
$$

Where $\rho$ is the density; $l$ and $d$ are the sample length and thickness, respectively; and $v$ is the eigenfrequency of the sample.

As is known, crystals are anisotropic. At the same time, the anisotropy of each individual single crystal is leveled due of their chaotic orientation in the ideal polycrystalline body. In practice, any external influence on the polycrystalline material (casting, pressure, heat treatment, etc) causes one or the other preferred orientation of the crystals (texture). Such polycrystalline material obtains anisotropy of properties and to some extent similar to the single crystal of corresponding symmetry. Thus, the anisotropy of properties (e.g., elasticity) of polycrystalline body is determined mainly by properties of its constituent crystallite, as well by distribution their over orientations in the polycrystalline sample i.e. by the texture. The stress state of metal grains may also have some influence on the anisotropy of properties of polycrystalline body. We will evaluate it later.

Earlier it was shown that texture is conveniently represented by integral characteristics of texture (ICT) (6) - (11) for the calculation of the anisotropy of the properties [9, 10]. These characteristics are integral convolutions of direction cosines defining orientation of individual crystallites in a space of a polycrystalline sample. The distribution function of the crystals orientation (ODF) plays a role of the weight function in the convolution integral.

$$
\begin{aligned}
& I_{1}=\left\langle\alpha_{13}^{2}\right\rangle, \\
& I_{2}=\left\langle\alpha_{23}^{2}\right\rangle, \\
& I_{3}=\left\langle\alpha_{33}^{2}\right\rangle, \\
& I_{4}=\left\langle\alpha_{13}^{4}\right\rangle, \\
& I_{5}=\left\langle\alpha_{33}^{4}\right\rangle, \\
& I_{6}=\left\langle\alpha_{13}^{2} \cdot \alpha_{23}^{2}\right\rangle,
\end{aligned}
$$

Where $\alpha_{i k}$ are cosines of the angles that set the orientation of the crystal relative to the coordinates of the sample; brackets denote averaging over all orientations of the crystals. We explore polycrystalline sheet material, which has an orthorhombic symmetry. Therefore, coordinate axes $\mathrm{X}, \mathrm{Y}, \mathrm{Z}$ of sample are directed along extrusion direction (ED), transverse direction (TD) and the normal direction (ND) to the sheet. The sum of the first three ICT from (6) - (11) is equal to one, as shown in $[9,10]$. Therefore, only five are independent variables.

Averaging (6)-(11) it may be performed with using the direct pole figure of the isotropic crystal plane. For hexagonal metal this may be PF $\{0002\}$. Then (6-11) takes the form [9,

$$
\text { 10]: } \begin{aligned}
I_{1} & =\frac{1}{2 \pi} \int_{0}^{\pi / 2} \int_{0}^{2 \pi} \sin ^{3} \alpha \cos ^{2} \beta P_{(0002)}(\alpha, \beta) d \alpha d \beta \\
I_{2} & =\frac{1}{2 \pi} \int_{0}^{\pi / 2} \int_{0}^{2 \pi} \sin ^{3} \alpha \sin ^{2} \beta P_{(0002)}(\alpha, \beta) d \alpha d \beta \\
I_{3} & =\frac{1}{2 \pi} \int_{0}^{\pi / 2} \int_{0}^{2 \pi} \sin \alpha \cos ^{2} \beta P_{(0002)}(\alpha, \beta) d \alpha d \beta
\end{aligned}
$$




$$
\begin{aligned}
& I_{4}=\frac{1}{2 \pi} \int_{0}^{\pi / 2} \int_{0}^{2 \pi} \sin ^{5} \alpha \cos ^{4} \beta P_{(0002)}(\alpha, \beta) d \alpha d \beta ;(15) \\
& I_{5}=\frac{1}{2 \pi} \int_{0}^{\pi / 2} \int_{0}^{2 \pi} \sin ^{5} \alpha \sin ^{4} \beta P_{(0002)}(\alpha, \beta) d \alpha d \beta ; \quad(16) \\
& I_{6}=\frac{1}{2 \pi} \int_{0}^{\pi / 2} \int_{0}^{2 \pi} \sin ^{5} \alpha \cos ^{2} \beta P_{(0002)}(\alpha, \beta) d \alpha d \beta .
\end{aligned}
$$

In relations (12) - (17) by means of $P_{(0002)}(\alpha, \beta)$ are denoted values of pole density on the pole figure (0002) in points with polar coordinates $(\alpha, \beta)$.

Using ICT and elastic compliances, the Young's modulus dependence on the direction in the plane of the sheet can be expressed as follows [10]:

$$
E(\gamma)=\left[S_{11}+2 d_{1} \Sigma_{1}(\gamma)+d_{2} \Sigma_{2}(\gamma)\right]^{-1} .
$$

In equation (18) we have identified

$$
\begin{aligned}
& \Sigma_{1}(\gamma)=I_{1} \cos ^{4} \gamma+I_{2} \sin ^{4} \gamma+\frac{1}{4}\left(I_{1}+I_{2}\right) \sin ^{2} 2 \gamma \\
& \Sigma_{2}(\gamma)=I_{6} \cos ^{4} \gamma+I_{4} \sin ^{4} \gamma+1.5 I_{5} \sin ^{2} 2 \gamma \\
& d_{1}=S_{13}-S_{11}+\frac{1}{2} S_{44} \\
& d_{2}=S_{11}+S_{33}-2 S_{13}-S_{44}
\end{aligned}
$$

Here $\gamma$ is the angle between ED and any other direction in the sheet plane.

The Young's modulus in a direction normal to the sheet is expressed by the relation

$$
\left(E_{N D}\right)^{-1}=S_{11}+d_{1} I_{3}++d_{2}\left(1-2 I_{1}-2 I_{2}+I_{4}+I_{5}+2 I_{6}\right)
$$

The dependence of the shear modulus from the direction in the plane of the sheet can be expressed as follows, using ICT and elastic compliances

$$
G(\gamma)=\left[d_{3}+d_{4} \Sigma_{1}(\gamma)+2 d_{2} \Sigma_{2}(\gamma)\right]^{-1} .
$$

In the last expression is indicated

$$
\begin{aligned}
& d_{3}=\frac{1}{2} S_{44}+S_{11}-S_{12} \\
& d_{4}=S_{11}-2 S_{33}-4 S_{13}-\frac{3}{2} S_{44}+S_{12}
\end{aligned}
$$

Functions $\Sigma_{1}(\gamma)$ and $\Sigma_{2}(\gamma)$ are depend only on the orientation of crystallites in a polycrystalline sample. The anisotropy of properties in the polycrystalline sheet plane depends in turn on values of above-mentioned functions in various directions. Therefore, the functions $\Sigma_{1}(\gamma)$ and $\Sigma_{2}(\gamma)$ can be named functions of elastic anisotropy.

The dependence of the Young's modulus from the direction in the sheet plane may be represented as a harmonic Fourier series with even harmonics as any periodic function [11]

$$
E^{-1}(\gamma)=F_{0}+F_{2} \cos 2 \gamma+F_{4} \cos 4 \gamma
$$

It was shown that $F_{0}, F_{2}, F_{4}$ can be represented by means of combinations of single-crystal elastic constants and ICT [12] 


$$
\begin{aligned}
& F_{0}=S_{11}+d_{1}\left(I_{1}+I_{2}\right)+\frac{3}{8} d_{2}\left(I_{4}+2 I_{5}+I_{6}\right) \\
& F_{2}=\frac{1}{2}\left[d_{1}\left(I_{1}-I_{2}\right)+d_{2}\left(I_{6}-I_{4}\right)\right] \\
& F_{4}=\frac{1}{8} d_{2}\left(I_{4}+I_{6}-6 I_{5}\right)
\end{aligned}
$$

It turns out that the elastic constant $S_{11}$ of single crystal and combinations $d_{1}$ and $d_{2}$ are expressed through ICT and coefficients of the Fourier series, if they have been early found from the experimental anisotropy of Young's modulus of polycrystalline sheet alloy, by means of next equations

$$
\begin{aligned}
& S_{11}=F_{0}-\frac{F_{2}\left(I_{1}+I_{2}\right)}{I_{1}-I_{2}}+4 \frac{F_{4}\left(I_{6}-I_{4}\right)\left(I_{1}+I_{2}\right)}{\left(I_{1}-I_{2}\right)\left(I_{4}+I_{6}-6 I_{5}\right)}-3 \frac{F_{4}\left(I_{4}+I_{6}+2 I_{5}\right)}{\left(I_{4}+I_{6}-6 I_{5}\right)} \\
& d_{1}=\frac{2 F_{2}}{I_{1}-I_{2}}-8 F_{4} \frac{I_{6}-I_{4}}{\left(I_{1}-I_{2}\right)\left(I_{4}+I_{6}-6 I_{5}\right)} \\
& d_{2}=\frac{8 F_{4}}{\left(I_{4}+I_{6}+6 I_{5}\right)}
\end{aligned}
$$

If experimentally measure anisotropy of the shear modulus in the plane of polycrystalline sheet alloy, then the remaining elastic constants of single crystal and their appropriate combinations that are represented by (25) - (26) may be expressed by other three equations similar to (28) (30). The joint solution of all these six equations is allowed would find all the desired elastic constants.

Above mentioned ICTs were calculated from (12-17) using data of the pole figure $\{0002\}$. The pole figure $\{0002\}$ was recorded on X-ray diffractometer DRON $3 \mathrm{M}$ in filtered Ka radiation. To the construction of complete pole figure we used a combination of methods on reflection and transmission [13].The normalization was performed by means of without texture sample, which was produced from fine recrystallized sawdust of investigated alloy.

\section{RESULTS AND DISCUSSION}

Figure 3 shows the PF $\{0002\}$ of sheet after extrusion of the alloy Mg-5\% (mass) Li.

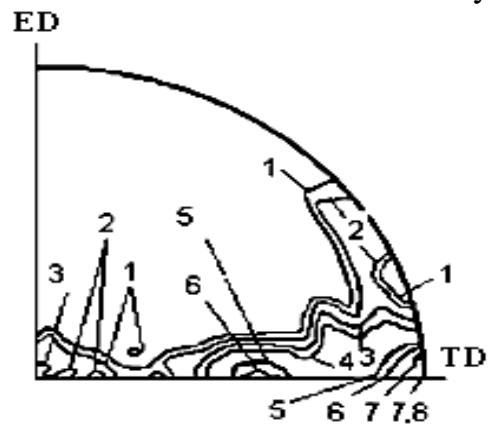

Figure 3. Pole figure $\{0002\}$ of extruded sheet of alloy $\mathrm{Mg}-5 \% \mathrm{Li}$

The texture is characterized by three maxima of pole density: in centre of PF (of 3.4), at the angular distances from $\mathrm{RD}$ in $55^{\circ}$ (of 6.4) and in $90^{\circ}$ (7.8). Texture of sheet alloy $\mathrm{Mg}-5 \% \mathrm{Li}$ (mass) (Figure 1) differs considerably from the rolling texture of central base type of pure 
magnesium [14]. However, similar textures previously were observed after quadratic and rectangular extrusion of magnesium [14].

Table 1 shows the ICT calculated from (12) - (17) and PF data (Figure 3).

Table 1. Integral characteristics of texture of alloy $\mathrm{Mg}-5 \%$ (mass) Li.

\begin{tabular}{|c|c|c|}
\hline \multicolumn{3}{|c|}{ ICT } \\
\hline $\mathrm{I}_{1}=0.063019$ & $\mathrm{I}_{3}=0.317952$ & $\mathrm{I}_{5}=0,490268$ \\
\hline $\mathrm{I}_{2}=0.619028$ & $\mathrm{I}_{4}=0.021757$ & $\mathrm{I}_{6}=0.031098$ \\
\hline
\end{tabular}

Let's calculate the anisotropy of Young's modulus of the sheet alloy Mg-5\% Li using the values of investigated alloy ICT (Table. 1) and single crystal elastic constants of pure magnesium (34) that we took from [15]:

$$
\left(S_{11}=2.21 ; d_{1}=0.31 ; d_{2}=-0.85\right) \cdot 10^{-11} \mathrm{~Pa}^{-1} .
$$

Combinations of elastic constants $d_{1}$ and $d_{2}$ in (34) were calculated from data [15] on formulas (21) - (22).

The Figure 4 shows the experimental anisotropy of Young's modulus in the sheet plane of investigated alloy as well as the anisotropy, calculated by means of ICTs (Table 1) and elastic constants (34) of pure magnesium, on the formula inverse to (18).

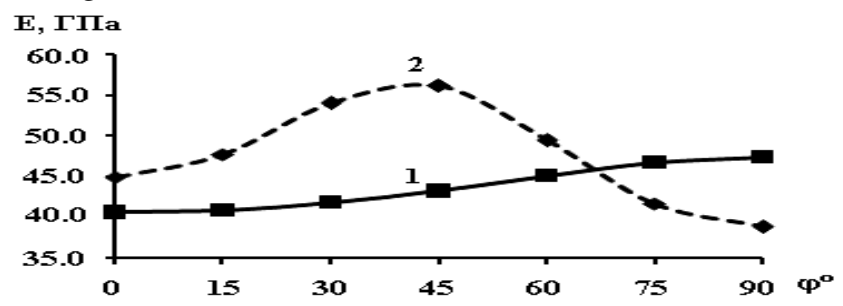

Figure 4. Anisotropy of Young's modulus in the sheet of the alloy $\mathrm{Mg}-5 \% \mathrm{Li}$. (1) is Experiment; (2) is calculation by the ICT (Table 1) and elastic constants (34) of pure magnesium Figure 4 shows that the theoretical curve is very different from the experimental anisotropy of Young's modulus of alloy sheet of $\mathrm{Mg}-5 \%$ Li not only quantitatively but and qualitatively. On the experimental curve a maximum is observed at an angle $90^{\circ}$ to the RD (i.e., in TD), while the calculated curve shows the maximum at the angle $45^{\circ}$ to the RD. Thus, the use of elastic constants of the single crystal $\mathrm{Mg}$ to calculate of the anisotropy of Young's modulus of alloy sheet of $\mathrm{Mg}-$ $5 \% \mathrm{Li}$ led to significant errors.

To calculate the single crystal elastic constant of $S_{11}$ and combinations of $d_{1}$ and $d_{2}$ for the investigated alloy, we in addition to ICT (Table 1) need to know Fourier coefficients of the expansion series of experimental anisotropy of Young's modulus in the sheet plane. As a result of our harmonic analysis of the experimental anisotropy of Young's modulus in the sheet plane of investigated alloy were obtained the following values of Fourier coefficients:

$$
F_{0}=2.30 \times 10^{-11} \mathrm{~Pa}^{-1} ; F_{2}=8.55 \times 10^{-13} \mathrm{~Pa}^{-1} ; F_{4}=-7.83 \times 10^{-14} \mathrm{~Pa}^{-1} .
$$

To determine how much correctly were received the Fourier coefficients (35), we compared the values of Young's modulus calculated by formula, inverse to (27) using the Fourier coefficients (35). Our estimates show that the calculated values of the Young's modulus of the investigated alloy are deviate from the corresponding experimental values insignificantly by no more than $4.5 \%$. 
New let's calculate by formulas (31) - (33) the elastic constant of $S_{11}$ and combinations $d_{1}$ and $d_{2}$ of investigated alloy using ICTs (Table. 1) and Fourier coefficients (35). As a result of corresponding calculations, were obtained the following values:

$$
\left(S_{11}=2.41 ; d_{1}=-0.307 ; d_{2}=-0.0209\right) \cdot 10^{-11} \mathrm{~Pa}^{-1} .
$$

In order to establish how much correctly we found the above mentioned elastic constants, we have re-calculated the anisotropy of Young's modulus in the sheet plane of investigated alloy with using our data (36). The comparison with the experiment had shown that deviations are not great namely not more than $5 \%$ (Figure 5).

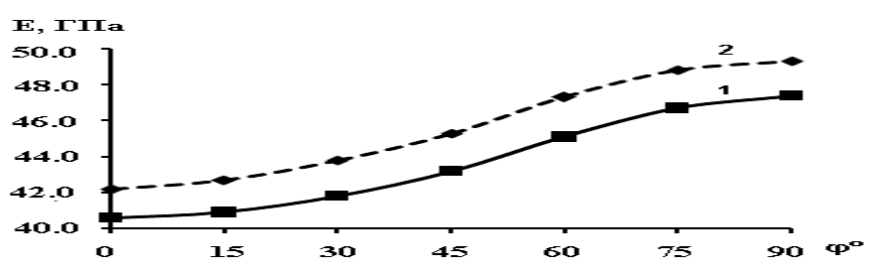

Figure 5. Anisotropy of Young's modulus in the sheet plane of the alloy $\mathrm{Mg}-5 \% \mathrm{Li}$. (1) is experiment; (2) is calculation by elastic constants of single crystal (36) of alloy $\mathrm{Mg}-5 \% \mathrm{Li}$

Now let's calculate the Young's modulus in a direction normal to the sheet plane $\left(E_{\mathrm{ND}}\right)$ of investigated alloy by formula, inverse to (23) using elastic constants (36) and ICTs (Table 1). As a result of calculation we have received $E_{\mathrm{ND}}=43.3 \mathrm{GPa}$.

Let's try to estimate remaining components of the elasticity tensor without information on the anisotropy of shear modulus in the sheet plane of investigated alloy. Note that for hexagonal metals, such as $\mathrm{Mg}$ component $S_{44}$ is approximately 12 times greater than $\left|S_{13}\right|$, and component $S_{11}$ is three times greater than $\left|S_{12}\right|[4,15]$. If take into account the above empirical relationships, then it is possible approximately to calculate all components of the elasticity tensor of investigated alloy:

$$
\left(S_{11}=2.41 ; S_{12}=-0.80 ; S_{13}=-0.43 ; S_{33}=1.77 ; S_{44}=5.05\right) \times 10^{-11} \mathrm{~Pa}^{-1} .
$$

The literature provides various estimates of the elastic constants of binary magnesium alloys with different content of lithium. For example, in [16] were calculated single crystal elastic constants $C_{\mathrm{ji}}$ of binary alloys of magnesium-lithium alloy with a different crystal structure (FCC, BCC, $\mathrm{HCP}$ ), in particular, for single crystals of hexagonal binary alloys magnesium alloys with lithium, silver, tin, indium. It is known that the maximum solubility at room temperature of lithium in the magnesium, when there remains almost exclusively HCP structure of the alloy, is about 5\% (mass). This corresponds to the contents approximately about 15 atomic $\%$ of lithium. The table 2 below shows some literary as well as our data of elastic constants $C_{i j}$, which were calculated from data of (37) according to formulas [3]. The Table 2 shows that values of $C_{i j}$ for magnesium alloy with about 15 atomic $\%$ of lithium content in the papers [16, 17] different more than twice for different constants. The Table 2 also shows that at the same time our elastic constants differ from those in the paper [17] less than $8 \%$ (for $C_{11}$ ) but not more than $30 \%$ (for $C_{44}$ ). 
Table 2. Elastic modules of single crystal of binary alloy Mg-Li.

\begin{tabular}{|c|c|c|c|c|c|}
\hline \multirow{2}{*}{ Alloy } & \multicolumn{5}{|c|}{ Elastic modules of single crystal of binary alloy } \\
& \multicolumn{5}{|c|}{ Mg-Li, GPa } \\
\cline { 2 - 6 } & $C_{11}$ & $C_{12}$ & $C_{13}$ & $C_{33}$ & $C_{44}$ \\
\hline $\mathrm{Mg}-15.0$ at. \% Li [16] & 92.0 & 10.0 & 5.0 & 103.0 & 42.0 \\
\hline $\mathrm{Mg}-15.94$ at. \% Li [17] & 55.49 & 24.15 & 20.22 & 57.42 & 15.25 \\
\hline $\begin{array}{c}\mathrm{Mg}-5 \text { (mass)\% Li } \\
\text { [in this article] }\end{array}$ & 51.2 & 20.1 & 17.1 & 64.7 & 19.8 \\
\hline
\end{tabular}

In the Introduction it was mentioned that a contribution to the Young's modulus and its anisotropy may make contribution the grain structure. Estimates of the grain structure effect in anisotropy of the properties previously were held by numerical simulation in [18]. It was shown that the error in the value of Young's modulus due to neglect of the grain structure was less than $0.3 \mathrm{GPa}$. For the Young's modulus of our investigated alloy $\mathrm{Mg}-5 \% \mathrm{Li}$ (mass) this error is approximately $0.7 \%$. It is within above mentioned range 5\% of deviation of the results of our calculations from experimentally measured values. Therefore, for quantitative calculations of elastic anisotropy polycrystalline material by means of corresponding single crystal elastic constants and integral characteristics of texture with precision up to $1 \%$ is possible not consider the impact of the grain structure of the metal that is also confirmed experimentally in [19].

The Figure 5 shows the anisotropy of Young's modulus of the investigated alloy after extrusion. The minimum value is observed in the extrusion direction, and maximum is in the transverse direction. The coefficient of anisotropy, which is the ratio of the difference between the maximum and the minimum value of the module to its minimum value, has enough significant value $16.8 \%$.

Description of texture using ICTs has such an advantage over other methods of description that allows you to assess the anisotropy of the properties and in the same time is less complex in comparison with the three-dimensional ODF. In addition, it would be possible to enter the texture certificate for metallic materials based on ICTs, as previously described in [5, 12].

\section{CONClusions}

Texture of sheet of magnesium alloy $\mathrm{Mg}-5 \%$ (mass.) $\mathrm{Li}$ after hot high pressure rectangular extrusion at $380^{\circ} \mathrm{C}$ is studied. Texture is characterized by spread of pole density from the center of pole figure $\{0002\}$ in the transverse direction on angles up to $90^{\circ}$. Corresponding integral characteristics of texture are calculated from pole figure $\{0002\}$. Integral characteristics of texture make up $I_{1}=0.063019 ; I_{2}=0.619028 ; I_{3}=0.317952 ; I_{4}=0.021757 ; I_{5}=0.490268 ; I_{6}=$ 0.031098. Integral characteristics of texture contain the necessary information to predict of tensor properties in any direction and can be recommended for certification of textured polycrystalline materials.

Use of single crystal elastic constants of magnesium for the calculation of anisotropy of Young's modulus in the plane of alloy sheet $\mathrm{Mg}-5 \% \mathrm{Li}$ (mass) leads to significant errors.

Integral characteristics of texture and Fourier coefficients of expansion series of experimental anisotropy of Young's modulus in the sheet plane of polycrystalline alloy enable count single crystal elastic constants or compliances of this alloy. Corresponding values of single crystal compliances of alloy $\mathrm{Mg}-5 \% \mathrm{Li}$ (mass) make up: $\left(\mathrm{S}_{11}=2.41 ; \mathrm{S}_{12}=-0.80 ; \mathrm{S}_{13}=-0.43 ; \mathrm{S}_{33}=1.77\right.$; $\left.\mathrm{S}_{44}=5.05\right) \times 10^{-11} \mathrm{~Pa}^{-1}$. 
We have calculated the anisotropy of Young's modulus in the sheet plane of polycrystalline alloy $\mathrm{Mg}-5 \% \mathrm{Li}$ using found single crystal constants. Deviation of calculated values from experimental is not significantly and did not exceed $5 \%$.

\section{REFERENCES}

[1] Leeflang, MA, Zhou, J \& Duszczyk, J. "Deformability and extrusion behaviour of magnesiumlithium binary alloys for bio-medical applications" (2009). In KU Kainer (Ed.), Magnesium, Proceedings of the 8th International Conference on Magnesium Alloys and their Applications. (pp. 1182-1188). Weinheim, Germany: Wiley-VCH. URL: http://www.3me.tudelft.nl/fileadmin/Faculteit/3mE/Over_de_faculteit/Afdelingen/Materials_Science

Engineering/MSE_News/scienceday2009/doc/M.A._Leeflang,_J._Zhou_and_J._Duszczyk.pdf

[2] Yang Z., Li J.P., Zhang J.X., Lorimer G.W. and J. Robson (2008) "Review on research and development of magnesium alloys", Acta Metall. Sin. (Engl. Lett.), Vol. 21, No. 5, pp. 313-328.

[3] Nye J.F. (1964) Physical Properties of Crystals, Oxford, Clarendon Press.

[4] Schulze G.E.R. (1974), Metallphysik, Wien, New York, Springer-Verlages und des Akademie Verlages, Berlin.

[5] San'kova S.V., Shkatulyak N.M., Usov V.V., Volchok N.A. "The Elastic Constants of the Single Crystal of the Mg-Zn-Zr-REM Alloy from the Data of the Elastic Anisotropy and the Texture of the Polycrystalline Sheet", Hindawi Publishing Corporation International, Journal of Metals, Vol. 2014, Article ID 142920, 6 pages http://dx.doi.org/10.1155/2014/142920

[6] Gall S., Mueller S., Reimers W. (2010), "Magnesium sheet production by using the extrusion process", Int. J. Mater. Forum, Vol. 3, Suppl. 1, p. 371-374.

[7] "Elastic moduli: overview and characterization methods". Technical Review ITC-ME/ATCP, URL: http://www.atcp-ndt.com/images/products/sonelastic/articles/RT03-TCP.pdf.

[8] Bunge H.-J. (1969), Mathematische Methoden der Texturanalyse, Berlin, Akademie-Verlag.

[9] Bryukhanov A.A., Gokhman A.R. (1985), "Integral characteristics of the texture of cubic and hexagonal metals", Izvestiya vuz. Fizika, No. 9, pp. 127-131 (Russian).

[10] Bryukhanov A.A. and Gokhman A.R., "Calculation method for determining of texture parameters of the tensor properties of cubic and hexagonal metals", Zavodskaya Laboratoriya, Vol. 53, No. 3, pp. 572-578, (1987) (Russian).

[11] Koutný F. (2006) "Elementary numerical methods \& Fourier analysis", URL: http://www.koutny- math.com/

[12] Bryukhanov A.A., Volchok N.A., and Sovkova T.S. (2010), "Effect of cold rolling on the characteristics of texture and anisotropy properties of alpha-alloy Ti-3Al-1,5V," // Materialy, No. 4, pp. 9-14 (in Russian).

[13] Wassermann G. and Greven J. (1962), “Texturen Metallischer Werkstoffe,” Springer, Berlin.

[14] Brokmeier H.-G., Randau C., Garbe U., Spalthoff P., Bohlen J. "Texture gradient in a bonelike extrusion profile of Mg Ze10" (2009), Proceedings of the 8th International Conference on Magnesium Alloys and their Applications, Wiley-VCH, 2009, pp. 583-588.

[15] Frederikse H.P.R., "Elastic constants of single crystals", URL: http://www.docstoc.com/docs/45454109/Elastic-Constants-of-Single-Crystals

[16] Phasha M.J., Ngoepe P.E., (2012) "An alternative DFT-based model for calculating structural and elastic properties of random binary HCP, FCC and BCC alloys: Mg-Li system as test case" Intermetallic, Vol. 21. pp. 88-96.

[17] Radwan Fae`q A.A (2011) "Some properties of magnesium alloys at different percentages of Lithium, Silver, Tin, Indium and Zinc", Advances in Applied Science Research, Vol. 2, No. 1, pp. 120-124.

[18] Kamaya M. (2009) “A procedure for estimating Young's modulus of textured polycrystalline materials", International Journal of Solids and Structures, Vol. 46, No. 13, pp. 2642-2649.

[19] Toonder J.M J., Dommelen J.A.W, and Baaijens F.P.T. (1999) "The relation between single crystal elasticity and the effective elastic behaviour of polycrystalline materials: theory, measurement and computation, "Modeling and Simulation in Materials Science and Engineering, Vol. 7, No. 6, pp. 909-928. 


\section{Authors}

\section{Short Biography}

Shkatulyak Natalia was born 17.05.1956, private- professor of physics of the South Ukrainian National Pedagogical University named after K.D. Ushinsky, Odessa. She holds a PhD in the field of solid state physics. The range of research interests are new substances and materials, texture, fractal nature of the structure and anisotropy of physical properties of crystalline solids and author of over 110 publications.

Usov Valentin was born 14.01.1950, Doctor of sciences in the field of physics of condensed state, Prof., Head of the department of technological and professional education of the South Ukrainian National Pedagogical University named after K.D. Ushinsky (Odessa), author of over 160 publications. The range of research interests are new substances and materials, texture, fractal nature of the structure and anisotropy of physical properties of crystalline solids and author of over 130 publications

Smirnova Svetlana was born 06.01.1985, Masters Degree, graduate student of Department of Physics of South Ukrainian National Pedagogical University named after K.D. Ushinsky, Odessa. The range of research interests are new substances and materials, texture, and anisotropy of physical properties of crystalline solids and author of 7 publications.
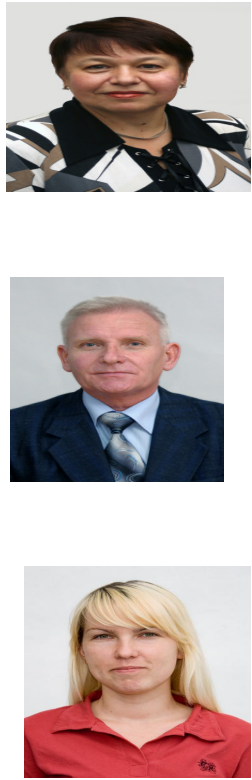